\title{
Ingrowing toenails in children and adolescents: is nail avulsion superior to nonoperative treatment?
}

\author{
Sumanth Kumar Gera' ${ }^{1}$, MBBS, MS, DK Halimatussadiah PG Zaini ${ }^{1}$, MBBS, Shiyao Wang ${ }^{1}$, MD, \\ Siti Hauzah Binte $\underline{\text { Abdul Rahaman }}{ }^{1}$, SRN, Rui Fang $\underline{\text { Chia }}^{1}$, DipBiotech, Kevin Boon Leong $\underline{\text { Lim }}^{1}$, FRCSEd, MBA
}

INTRODUCTION Ingrowing toenail (IGTN) or onychocryptosis is not uncommon in children and adolescents. However, there is a dearth of evidence in the literature on the management of IGTN in this age group. This study aimed to compare the results of nonoperative treatment for IGTN with that of operative treatment among children and adolescents.

METHODS All children and adolescents who were treated for IGTN at our institution between 2010 and 2014 were included for this retrospective study. Demographic data, treatment prescribed and outcome at six months after presentation were analysed.

RESULTS Overall, 199 patients were recruited. There were $123(61.8 \%)$ boys and $76(38.2 \%)$ girls. Median age was 14 years. Among 199 toes, 162 (81.4\%) were treated nonoperatively, with nail care advice, topical antibiotics and daily cleansing. Only $37(18.6 \%)$ toes were treated operatively. In the operative group, $23(62.2 \%)$ patients underwent wedge resections, while the remaining $14(37.8 \%)$ had total nail avulsions; for all patients, germinal matrices were preserved. At the six-month follow-up, there were $5(3.1 \%)$ cases of recurrence in the nonoperative group when compared to $3(8.1 \%)$ recurrences in the operative group.

CONCLUSION We recommend that IGTN in children and adolescents be treated in the first instance by nonoperative methods. Operative options can be considered for resistant cases or in case of recurrence of IGTN.

Keywords: adolescents, ingrown toenails, nail avulsion, nail care

\section{INTRODUCTION}

An ingrown toenail, also known as onychocryptosis, occurs when the nail plate grows into the adjacent periungual skin and acts as a foreign body, causing inflammation and infection..$^{(1,2)}$ It can affect any toe, but the big toe nail is the most commonly affected. ${ }^{(3)}$

While ingrowing toenails (IGTNs) are common, its incidence in Singapore among children and adolescents is unknown. The highest incidence occurs in teenagers and adults aged 11-30 years. ${ }^{(4)}$ While the aetiology of IGTN is not fully understood, contributing factors include genetic susceptibility, trauma, tight-fitting shoes and incorrect trimming of toenails. ${ }^{(5-8)}$

The disease process in IGTN comprises three stages: $:^{(5,6,9,10)}$ Stage 1 (inflammation), or the mildest form, which consists of swelling, erythema, oedema and swelling of the nail fold, and pain with pressure; Stage 2 (infection), or further swelling associated with seropurulent drainage, infection and ulceration of the nail fold; and Stage 3 (granulation), or the most severe disease stage, which consists of chronic inflammation and granulation.

Treatment of IGTN is indicated when there is significant pain or infection, or chronic recurrent paronychia. ${ }^{(3)}$ The pain can become so severe that it may be disabling, affecting one's daily activities, such as school, work and physical activities. There are nonoperative and operative treatment options, and the decision for one option type over the other depends largely on the stage at presentation as well as patient preference.

Nonoperative treatment consists of soaking the affected toe in warm soapy water for ten minutes followed by the application of a topical antibiotic ointment to the affected nail fold at least twice a day. ${ }^{(11)}$ Patients and caregivers are taught nail care tips and techniques, including the avoidance of tight-fitting footwear and overzealous nail trimming. They are advised to cut the nail such that the distal corners of the nail are visible and distinct, rather than attempting to shape them into a smooth round edge and risk leaving a sharp spike of nail at either the medial or lateral corner. Nonoperative management for IGTN is often recommended for Stage 1 and 2 diseases. It is reiterated to patients that treatment success is to a certain extent dependent upon compliance with prescribed treatment, and that clinical improvement may not be immediately obvious.

Operative treatment options are mainly reserved for Stage 3 disease or those for whom nonoperative treatment has not been successful. ${ }^{(12)}$ Common operative options in children and teenagers include complete or partial nail avulsion alone, or with associated germinal matrix ablation. ${ }^{(13-15)}$ Various forms of anaesthesia are available, and this determines whether the procedure can be done as an outpatient procedure, day surgery procedure or whether admission to hospital is required. Relief of symptoms is usually immediate. However, most often, the new nail that grows is thick and less pliant. Nail plate avulsion without germinal matrix ablation also has a high rate of recurrence. ${ }^{(12,16)}$ It has been reported that some patients have to undergo operative treatment six times after the index procedure. ${ }^{(17)}$

To the best of our knowledge, there is a dearth of evidence in the literature with regard to the treatment of IGTN in children

1Department of Orthopaedic Surgery, KK Women's and Children's Hospital, Singapore

Correspondence: Dr Sumanth Kumar Gera, Senior Staff Registrar, Department of Orthopaedic Surgery, KK Women's and Children's Hospital, 100 Bukit Timah Road, Singapore 229899. Sumanth.Kumar@kkh.com.sg 
and adolescents. This retrospective study aimed to compare the outcomes of nonoperative treatment and nail plate avulsion without matrix ablation for IGTN.

\section{METHODS}

This study, which was conducted at a tertiary care children's hospital in Singapore, was approved by the institutional review board. Clinical notes of the patients who presented to our clinic between 2010 and 2014 were reviewed and included for study if they satisfied the inclusion criteria, which were: (a) all children and adolescents with a diagnosis of IGTN (Stage 2 or 3); and (b) age up to 16 years. Patients were excluded if they had the following conditions: Stage 1 IGTN; concurrent toe deformity; or any underlying condition (e.g. psoriasis and diabetes mellitus).

Patient's age, symptomology, disease stage and treatment prescribed at presentation, and the time taken for complete resolution of signs and symptoms were recorded. Any recurrence after the initial treatment was also noted. Data was analysed for two treatment groups - nonoperative and operative. Details of nonoperative and operative treatment options were discussed with patient and family, so that they could then make an informed choice.

All patients who opted for nonoperative treatment were taught good nail care practices by our outpatient clinic nurses. They were prescribed antiseptic soap solution, topical antibiotics and a course of oral antibiotics in severe cases of IGTN. These patients were subsequently referred to a podiatrist. Patients were also given an information booklet on IGTN. In addition, all patients were given a doctor's note to excuse them from physical activities and wearing covered shoes. They were subsequently followed up at 4-6 weeks, three months and six months from the index clinic visit.

Operative treatment was performed as a day surgery procedure, either under local or regional anaesthesia. Postoperatively, patients were followed up at one week for wound inspection, and then at 4-6 weeks, three months and six months thereafter.

Fisher's exact test was used to determine significant differences in the rates of symptom resolution following operative and nonoperative treatments. There were three possible outcomes: (a) early findings at 4-6 weeks after initial treatment; (b) findings at three months; or (c) findings at six months. Fisher's exact test was used to test the hypothesis: $\mathrm{H} 0: \pi=0.50$ versus $\mathrm{H} 1: \pi \neq 0.50$ for each of the three outcomes possible, where $\pi$ was the true proportion of patients exhibiting a particular outcome. The significance level was set at $\alpha=0.05$. All analyses were performed using IBM SPSS Statistics version 19.0 (IBM Corp, Armonk, NY, USA).

\section{RESULTS}

Overall, 286 patients presented to our outpatient clinic with IGTN during the study period. Of these, 199 patients met the inclusion criteria. The remaining 87 were excluded for other reasons, such as no IGTN ( $n=14)$, IGTN on other toes instead of the big toe $(n=3)$, refused treatment $(n=3)$ and limited follow-up $(n=67)$.

Among the 199 patients who were included for the study, there were $123(61.8 \%)$ boys and 76 (38.2\%) girls. Median age
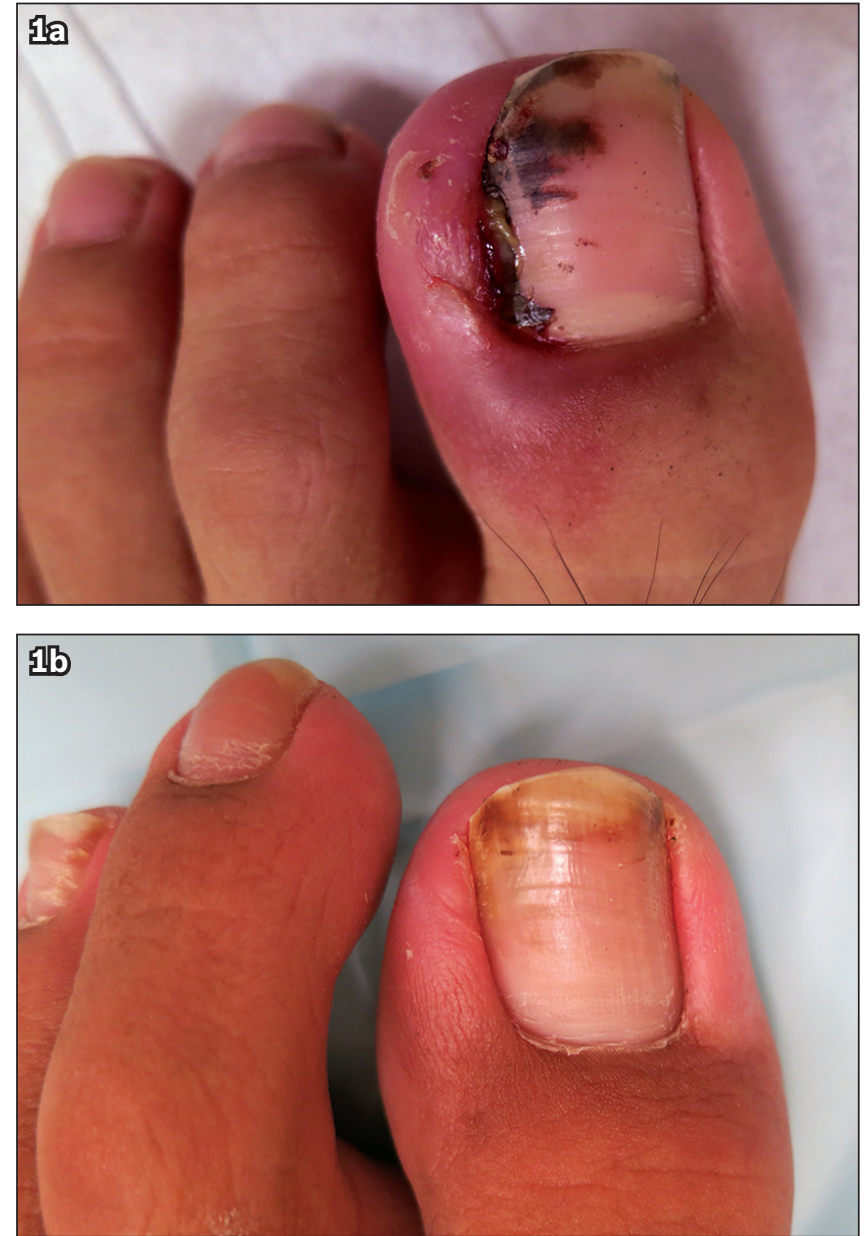

Fig. 1 Photographs show an ingrowing toenail (a) before and (b) after initiating nonoperative treatment with nail care.

was 14 years. Among them, 162 of 199 (81.4\%) toes were treated nonoperatively with nail care advice, topical antibiotics and daily cleansing (Fig. 1) and 37 (18.6\%) toes were treated operatively. In the operative group, $23(62.2 \%)$ toes underwent wedge resections without matrix ablation, while the remaining 14 (37.8\%) had total nail avulsions without matrix ablation.

In the nonoperative group, 141 of $162(87.0 \%)$ patients reported resolution of symptoms while the remaining 21 (13.0\%) patients continued to be symptomatic at the 4-6-week follow-up. In the operative group, 36 of 37 (97.3\%) patients experienced complete resolution of symptoms, while 1 (2.7\%) patient had persistent symptoms at the $4-6$-week follow-up. These results suggested that surgery as the initial treatment allowed faster resolution of IGTN when compared with nonoperative approaches. However, this difference was not statistically significant ( $p=0.085)$ (Table I).

All patients with persistent symptoms at the 4-6-week followup had recovered completely at the three-month follow-up and were discharged. At the six-month follow-up, 8 (4.0\%) patients in total (nonoperative group, $\mathrm{n}=5[3.1 \%]$; operative group, $\mathrm{n}=3$ $[8.1 \%]$ ) were found to have recurrence of symptoms (Table II). All other patients who were discharged with no issues or had defaulted follow-up at six months were considered as resolved at six months. 
Table I. Findings at the 4-6-week follow-up.

\begin{tabular}{|lllll|}
\hline \multirow{2}{*}{ Treatment arm } & \multicolumn{2}{c}{ No. of patients } & Resolution rate (\%) & p-value* \\
\cline { 2 - 3 } & Resolution of signs and symptoms & Persistence of signs and symptoms & \\
\hline Nonoperative $(n=162)$ & 141 & 21 & 87.0 & \\
\hline Operative $(n=37)$ & 36 & 1 & 97.3 & \\
\hline Total $(n=199)$ & 177 & 22 & 88.9 & 0.085 \\
\hline
\end{tabular}

*Fisher's exact test.

Table II. Findings at the six-month follow-up.

\begin{tabular}{|llll|}
\hline \multirow{2}{*}{ Treatment arm } & \multicolumn{2}{c}{ No. of patients } & Recurrence rate (\%) \\
\cline { 2 - 4 } & Recurrence & No recurrence & \\
\hline Nonoperative $(n=162)$ & 5 & 157 & 3.1 \\
\hline Operative $(n=37)$ & 3 & 34 & 8.1 \\
\hline Total $(n=199)$ & 8 & 191 & 4.0 \\
\hline
\end{tabular}

\section{DISCUSSION}

IGTN is relatively common among children and adolescents. About 15-20 cases of IGTN are seen at our clinic each month. In the first instance, nonoperative measures are generally recommended for Stages 1 and 2 of the disease, while operative options are recommended for patients with Stage 3 disease.

In our study, the odds of symptom resolution at 4-6 weeks after treatment were found to be five times higher with operative treatment than with nonoperative treatment. This result was in agreement with studies on adult patients with IGTN that found that there is a better chance of symptom resolution if treated with surgical methods instead of non-surgical methods. ${ }^{(18)}$ However, the difference was not statistically significant between the two treatment arms $(p=0.085)$, and so there was insufficient evidence to conclude that nonoperative methods were more effective than nail avulsion for IGTN.

At the three-month follow-up, both operative and nonoperative groups showed complete resolution of signs and symptoms. However, 8 (4.0\%) patients (nonoperative group, $\mathrm{n}=5[3.1 \%]$; operative group, $\mathrm{n}=3[8.1 \%]$ ) experienced recurrence of symptoms at six months. These findings mirrored those of a study by Lazar et al, ${ }^{(19)}$ where excellent outcomes were reported for 20 paediatric patients. Furthermore, studies have also reported that operative treatment carried significant morbidity, inconvenience and most of all, unacceptably high recurrence rates among children. ${ }^{(17,19)}$

We found that, among patients with Stage 2 and Stage 3 IGTN, a majority $(81.4 \%)$ of patients did not require surgery and recovered well at six months, while those who underwent surgery had a recurrence rate of $8.1 \%$ at six months. However, patients who opted for operative treatment in our study had their germinal matrix preserved, which might explain the recurrence rate among these patients. Also, patients were not randomised into the two treatment groups in our study, limiting the comparability and generalisability of our findings. The retrospective nature of the study and its small sample size were other limitations.

In conclusion, we found that Stage 2 and Stage 3 IGTN in children and adolescents may be treated in the first instance by nonoperative methods. Operative options could be considered for resistant cases or in case of recurrence of IGTN.

\section{ACKNOWLEDGEMENTS}

This work is dedicated to the memory of Dr Winnie Fung, our friend and colleague. We would also like to thank Ms Frances Lim, Associate Executive, Department of Orthopaedic Surgery, for her administrative support.

\section{REFERENCES}

1. Reyzelman AM, Trombello KA, Vayser DJ, Armstrong DG, Harkless LB. Are antibiotics necessary in the treatment of locally infected ingrown toenails? Arch Fam Med 2000; 9:930-2.

2. DeLauro NM, DeLauro MT. Onychocryptosis. Clin Podiatr Med Surg 2004; 21:617-30

3. Heidelbaugh J, Lee H. Management of the ingrown toenail. Am Fam Physician 2009; 79:303-8.

4. Keeman JN. [The ingrown nail]. Ned Tijdschr Geneeskd 1986; 130:1431-4. Dutch.

5. Zuber TJ. Ingrown toenail removal. Am Fam Physician 2002; 65:2547-52, 2554

6. Kaleel SS, Iqbal S, Arbuthnot J, Lamont G. Surgical options in the management of ingrown toenails in paediatric age group. Foot 2007; 17:214-7.

7. Yang G, Yanchar NL, Lo AY, Jones SA. Treatment of ingrown toenails in the pediatric population. J Pediatr Surg 2008; 43:931-5.

8. Mitchell S, Jackson CR, Wilson-Storey D. Surgical treatment of ingrown toenails in children: what is best practice? Ann R Coll Surg Engl 2011; 93:99-102.

9. Aksakal AB, Atahan C, Oztaş P, Oruk S. Minimizing postoperative drainage with $20 \%$ ferric chloride after chemical matricectomy with phenol. Dermatol Surg 2001; 27:158-60.

10. Ozdemir E, Bostanci S, Ekmekci P, Gurgey E. Chemical matricectomy with $10 \%$ sodium hydroxide for treatment of ingrowing toenails. Dermatol Surg 2004; 30:26-31.

11. Daniel CR 3rd, lorizzo M, Tosti A, Piraccini BM. Ingrown toenails. Cutis 2006; 78:407-8.

12. Freiberg A, Dougherty S. A review of management of ingrown toenails and onychogryphosis. Can Fam Physician 1988; 34:2675-81.

13. Zadik FR. Obliteration of the nail bed of the great toe without shortening the terminal phalanx. Bone Joint J 1950; 32:66-7.

14. Ross WR. Treatment of the ingrown toenail and a new anesthetic method. Surg Clin North Am 1969; 49:1499-504.

15. Winograd AM. A modification in the technic of operation for ingrown toe-nail. 1929. J Am Podiatr Med Assoc 2007; 97:274-7.

16. Haneke E. Controversies in the treatment of ingrown nails. Dermatol Res Pract 2012; 2012:783924.

17. Thompson TC, Terwilliger $\mathrm{C}$. The terminal syme operation for ingrown toenail. Surg Clin North Am 1950; 31:575-84.

18. Espensen $\mathrm{EH}, \mathrm{Nixon} \mathrm{BP}$, Armstrong DG. Chemical matrixectomy for ingrown toenails: is there an evidence basis to guide therapy? J Am Podiatr Med Assoc 2002; 92:287-95

19. Lazar L, Erez I, Katz S. A conservative treatment for ingrown toenails in Children. Pediatr Surg Int 1999; 15:121-2. 